\title{
POTENCIAL DE ALMACENAMIENTO DE SEMILLAS DE Genipa americana L. EN EL DEPARTAMENTO DEL QUINDÍO
}

\section{STORAGE POTENTIAL OF SEEDS OF Genipa americana $L$. IN THE DEPARTMENT OF QUINDÍO}

\author{
Sandra Viviana Ramírez Morales ${ }^{1} \&$ Andrés Felipe Orozco Cardona ${ }^{2}$ \\ 1. Centro de Estudios e Investigaciones en Biodiversidad y Biotecnología (CIBUQ) \\ 2. Docente Programa de Biología, Investigador Centro de Estudios e Investigaciones en Biodiversidad y Biotecnología (CIBUQ), Universidad del \\ Quindío.
}

Fecha de recibido: Febrero 3 de 2010

Fecha de aceptado: Junio 9 de 2010

Correspondencia: Programa de Biología, Universidad del Quindío, Av. Bolívar calle 12 norte Armenia Quindío. Correo electrónico: andresorozco@uniquindio.edu.co

\section{RESUMEN}

Se evaluó la eficiencia del método de almacenamiento para la conservación de semillas de Genipa americana procedentes de 6 poblaciones de la zona cálida del departamento del Quindío. El estudio se desarrolló en el laboratorio de Biotecnología del Centro de Estudios e Investigaciones de Biodiversidad y Biotecnología de la Universidad del Quindío (CIBUQ). Se siguió la metodología sugerida por Bioversity International para el manejo de semillas en bancos de germoplasma, con el fin de establecer un protocolo de almacenamiento para la conservación a corto plazo de semillas de la especie a través de la determinación de la temperatura y el sustrato de almacenamiento adecuado. Las pruebas iníciales revelaron $75 \%$ de viabilidad, 43\% de contenido de humedad, 78\% de porcentaje de germinación, índice de velocidad de germinación de 2 semillas por día y 23 días en tasa de germinación. El potencial germinativo de la especie puede mantenerse a corto plazo en almacenamiento a temperatura ambiente, utilizando torlita para controlar el contenido de humedad, el cual debe estar por encima del $10 \%$ para conservar su viabilidad. Los resultados obtenidos demuestran que el comportamiento de almacenamiento de las semillas de G. americana corresponde al de una semilla tipo intermedia.

Palabras claves: Conservación, semilla intermedia, viabilidad, germinación, contenido de humedad.

\section{ABSTRACT}

A method of storage for conservation of Genipa americana seeds was evaluated. Seeds were from 6 populations located in the entire warm area of Quindio. The study was conducted in the laboratory of Biotechnology, Centre for Studies and Research on Biodiversity and Biotechnology at the University of Quindio (CIBUQ). A methodology suggested by Bioversity International for handling seeds in gene banks was used in order to establishing a storage protocol for short-term conservation of seeds of the species through the determination of temperature and substrate proper to storage. Initial tests revealed $75 \%$ viability, $43 \%$ moisture content, $78 \%$ germination percentage, germination speed index of 2 seeds per day and 23 days for germination rate. The potential of germination of Genipa americana can keep itself to short-term in storage at room temperature, using torlita to control the moisture content which should be only up to $10 \%$ for keeping its viability. The results obtained show that the storage behavior of Genipa americana seeds is similar to any type of intermediate seed.

\section{INTRODUCCIÓN}

En la actualidad la flora de los bosques tropicales del mundo está siendo destruida a un ritmo acelerado y numerosas especies están al borde de la extinción. La diversidad de especies de estos bosques se refleja en una notable variedad de semillas; las cuales presentan viabilidad variable $y$ longevidad promedio corta ${ }^{(1)}$.

El almacenamiento de semillas permite la conservación del germoplasma de plantas valiosas y en peligro de extinción ${ }^{(2)}$. Sin embargo, los bancos de semillas almacenan en su mayoría muestras de plantas cultivadas.

Las especies forestales de los ecosistemas del Quindío, tienen un valor socio-económico y ecológico intrínseco; sin embargo las poblaciones de varias de éstas se encuentran diezmadas.

Entre estas especies se encuentra Genipa americana, conocida comúnmente como Jagua, la cual es un árbol de la familia Rubiaceae ${ }^{(3)}$ que crece en ecosistemas húmedos tropicales de Colombia ${ }^{(4)}$. Tiene gran valor alimenticio, medicinal, industrial y ecológico. Además, ha sido utilizada como especie promisoria en modelos de recuperación de áreas degradadas en ambientes del Estado de São Paulo ${ }^{(5)}$. Se distribuye desde México a través de América Central, las Antillas y norte de América del Sur hasta Paraguay y norte de Argentina. Fructifica durante todo el año y un mismo 
individuo puede poseer frutos en todos los estados de crecimiento por la periodicidad en la floración y la larga durabilidad de los frutos ${ }^{(6-7)}$; los cuales tardan hasta un año en madurar ${ }^{(8)}$.

Para la especie se han realizado estudios sobre morfofisiología, distribución, usos, fenología en Colombia y algunos ensayos de germinación y almacenamiento en Brasil.

Por lo anterior el objetivo de esta investigación fue evaluar el potencial de almacenamiento de semillas de $G$. americana del departamento del Quindío.

\section{METODOLOGÍA}

\section{Área de estudio}

El estudio se realizó en la zona cálida del departamento del Quindío, entre los 900 y los 1100 msnm, en el Valle del río la Vieja (Figura 1).

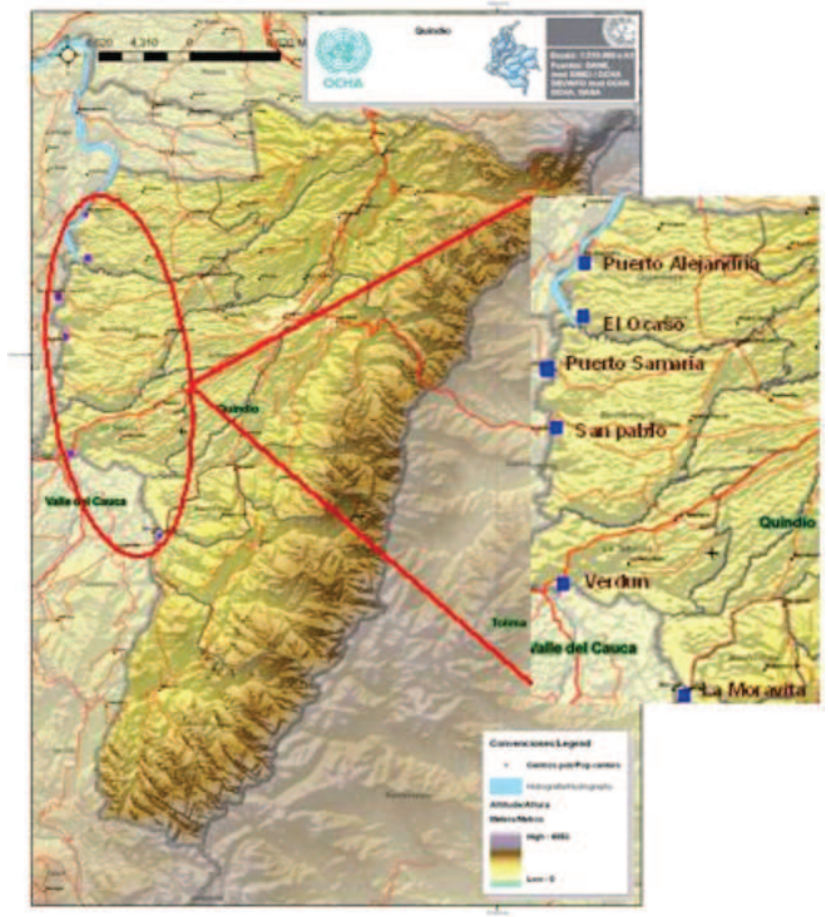

Figura 1. Sitios de muestreo de Genipa americana en el departamento del Quindío representados por cuadros de color azul (9)

Los sitios de muestreo correspondieron a: Reserva La Montaña del Ocaso, Vereda El Laurel y Vereda Puerto Alejandría, en el municipio de Quimbaya; Vereda Puerto Samaria y Vereda San Pablo, en el municipio de Montenegro; Vereda La Moravita en el municipio de Pijao y un último sitio localizado en la Vereda El Verdún, municipio de Caicedonia, Valle del Cauca. La precipitación pluvial anual de la zona está entre los 1000-2000msnm y la temperatura media anual entre 18-24으.

\section{Procedimientos}

El trabajo se llevó a cabo en dos fases:
Fase de Campo:

Previo a la colecta de los frutos se revisó el material del Herbario Universidad del Quindío (HUQ), para ubicar los sitios de muestreo en el departamento; luego se hizo un premuestreo para identificar fuentes semilleras y realizar un seguimiento del estado de madurez de los frutos a colectar.

Se colectaron frutos maduros de individuos de la especie, que estuviesen en fructificación, entre febrero y abril de 2009. De cada procedencia se colectaron 20 frutos maduros que no presentaran señales de deterioro. La colecta se hizo directamente del árbol con un cortarramas; de cada sitio se muestrearon 1 o 2 individuos, por encontrarse pocos árboles semilleros y la mayoría con frutos inmaduros. Para cada individuo se registró el diámetro y la altura y se tomaron las coordenadas geográficas del sitio de muestreo con un GPS para tener ubicados los árboles semilleros.

Para el transporte del material colectado al sitio de procesamiento se utilizaron bolsas plásticas con aberturas laterales, para permitir una buena ventilación de los frutos.

\section{Fase de laboratorio}

\section{- Presecado ylimpieza}

El material se ingresó al laboratorio de Biotecnología de la Universidad del Quindío, donde se extrajeron las semillas y se realizó su limpieza, retirando el material que no hiciera parte de éstas e inspeccionándolas para detectar lesiones internas y excluir las infestadas o deterioradas. Posteriormente se secaron por 3 horas a la sombra, sobre papel absorbente, donde circulara el aire y evitando el calor (10).

- Evaluación inicial de la calidad Las pruebas de calidad se realizaron de acuerdo a los protocolos establecidos por Rao et al. (2007) que se mencionan a continuación:

\section{Valoración del contenido de humedad}

Para esta prueba se tomaron 40 semillas de cada procedencia, cada grupo de 40 semillas se dividió en dos partes iguales, las cuales fueron secadas por dos métodos diferentes: Sílica gel y torlita. Las semillas se introdujeron en frascos de vidrio con selle hermético que contenían en el fondo sílica gel autoindicador azul o torlita, según el caso. El peso del gel de sílice utilizado fue igual al de las semillas (10) y la cantidad de torlita correspondió al volumen que ocuparon las semillas.

El contenido de humedad $(\mathrm{CH})$ se determinó pesando las semillas en una balanza digital Professional-Scale LT Series con una precisión de $0.1 \mathrm{~g}$ y capacidad de $500 \mathrm{~g}$. Se tomaron datos de peso desde 0 hasta 72 horas.

\section{Viabilidad}

La estimación del porcentaje de viabilidad (PV) de las

Rev. Invest. Univ. Quindío (21): 143 - 152. Armenia - Colombia 
semillas de G. americana se realizó con muestras de 30 semillas de cada sitio de procedencia. Se utilizó la prueba topográfica de tetrazolio al $1 \%$ en oscuridad a temperatura ambiente durante 24 horas; previamente las semillas se hidrataron durante 48 horas. Para facilitar la interpretación de la tinción, las semillas se clasificaron en tres categorías (Tabla 1).

Tabla 1. Categorías de viabilidad de las semillas de G. americana según el patrón de tinción

\begin{tabular}{|c|c|c|}
\hline Categoria & Color & $\begin{array}{l}\text { Porcentaje de } \\
\text { Viabilidad }\end{array}$ \\
\hline $\begin{array}{l}\text { I. Semillas totalmente teñidas } \\
\text { que son viables. }\end{array}$ & & $=75 \%$ \\
\hline II. Semillas parcialmente viables. & & $50-74 \%$ \\
\hline $\begin{array}{l}\text { III. Semillas libres de coloración } \\
\text { que no son viables. }\end{array}$ & & $<50 \%$ \\
\hline
\end{tabular}

\section{Germinación}

Se tomó al azar una muestra de 60 semillas de cada procedencia. Previo a la germinación las semillas se desinfectaron con Tween 20alcohol comercial-hipoclorito de sodio comercial y se sembraron en cajas petri sobre papel secante humedecido con agua destilada. Se mantuvieron a temperatura ambiente con 12 horas luz durante 33 días. Cada 3 o 4 días el sustrato se roció con 12 gotas de agua destilada y se hicieron los respectivos conteos del número de semillas germinadas por día, basado en una longitud de la radícula superior a $0.5 \mathrm{~cm}$. Se calculó el porcentaje de germinación (PG), la tasa de germinación (TG) y el índice de velocidad de germinación (IVG) $^{(11)}$.

\section{Secado de las semillas y almacenamiento}

Previo al almacenamiento, las semillas se dispusieron sobre papel secante al interior de una cabina desecadora con sílica gel como agente absorbente durante un periodo de 48 horas, lapso de tiempo en el cual las semillas alcanzan un rango de $\mathrm{CH}$ de humedad entre $10-13 \%$ determinado previamente en la prueba de humedad inicial de las semillas.

Las semillas secadas fueron pesadas en grupos de 150 y se introdujeron en bolsas ziploc de $14 \times 10 \mathrm{~cm}$, a las cuales previamente se les añadieron cantidades de sílica gel o torlita. Posteriormente las bolsas se ubicaron al interior de recipientes de vidrio transparente con selle hermético, según lo recomendando por $^{(12-13)}$ y se conservaron a 3 temperaturas diferentes.

\section{Pruebas de almacenamiento}

Para evaluar el potencial de almacenamiento se utilizó un diseño experimental de bloques al azar en un arreglo factorial con 3 factores controlados:

- Temperatura de almacenamiento con 3 niveles ( 5 으, $-20^{\circ} \mathrm{C}$ y temperatura ambiente).
- Tiempo de almacenamiento con 4 niveles: Prealmacenamiento, 30 días, 60 días y 90 días.

- Sustrato de almacenamiento con 2 niveles: Sílica gel y Torlita.

Se realizaron en total 24 tratamientos y las variables de respuesta fueron las pruebas de viabilidad, germinación y $\mathrm{CH}$. La unidad muestral fue 22 semillas por tratamiento (10 en germinación, 5 en viabilidad y 7 en $\mathrm{CH}$ ). Se utilizaron 2376 semillas en las pruebas de calidad durante el almacenamiento y 780 en las pruebas de calidad iniciales, para un total de 3156 semillas.

Cada 30 días se realizaron las pruebas anteriormente descritas para verificar la calidad de las semillas conservadas. Este procedimiento se repitió en 3 oportunidades, es decir hasta los 90 días de almacenamiento. Para los ensayos de germinación, se aplicaron 3$5 \mathrm{~g}$ del fungicida Vitavax 300 disuelto en agua destilada debido a la alta incidencia de contaminación fúngica. Así mismo cada semana se verificó visualmente que la sílica gel al interior de los recipientes herméticos presentara color azul; en caso contrario, la sílica rosada se reemplazó.

\section{Análisis estadístico}

Se construyeron matrices de datos para las variables de respuesta. Para cada matriz de datos se hizo un análisis de varianza (ANOVA) empleando el paquete estadístico Statistix versión 9.0 con el fin de determinar diferencias significativas para los factores e interacciones entre ellos con un nivel de significancia del 95\%. Los resultados de la PG fueron transformados en arco seno previo al tratamiento estadístico, por análisis de varianza. El análisis de la TG se basó en los datos transformados al logaritmo natural y el IVG a la raíz cuadrada, con la finalidad de establecer homogeneidad de varianzas. Para determinar cuál de los tratamientos significativos era el más adecuado se aplicó la prueba de comparaciones LSD hallando la Diferencia Mínima Significativa (DMS). Finalmente, se hicieron gráficas mediante el programa Matlab (Versión 7.0) para mostrar las interacciones de segundo orden entre los factores, para los resultados de la germinación, viabilidad y contenido de humedad.

\section{Evaluación inicial de calidad Contenido de Humedad:}

El CH inicial de las semillas fue de aproximadamente $43 \%$ (Figura 2). El método de sílica gel puede reducir el $\mathrm{CH}$ hasta aproximadamente un $9 \%$ durante un periodo de 72 horas (Figura $2 a$ y $2 c$ ), mientras que con el método de torlita solo se logró reducir hasta un $28 \%$ en promedio (Figura 2 b y 2 c).

La reducción en el $\mathrm{CH}$ obtenida por ambos métodos de secado corrobora que la sílica gel reduce la humedad a valores inferiores a los iniciales en contraste con la torlita, que retiene menos la humedad.

Además, el $\mathrm{CH}$ inicial se encuentra dentro del rango reportado por la especie ${ }^{(14)}$.

A si mismo se ha reportado que la humedad inicial de las semillas de G. americana está directamente relacionada al grado de maduración de los frutos ${ }^{(15)}$. 

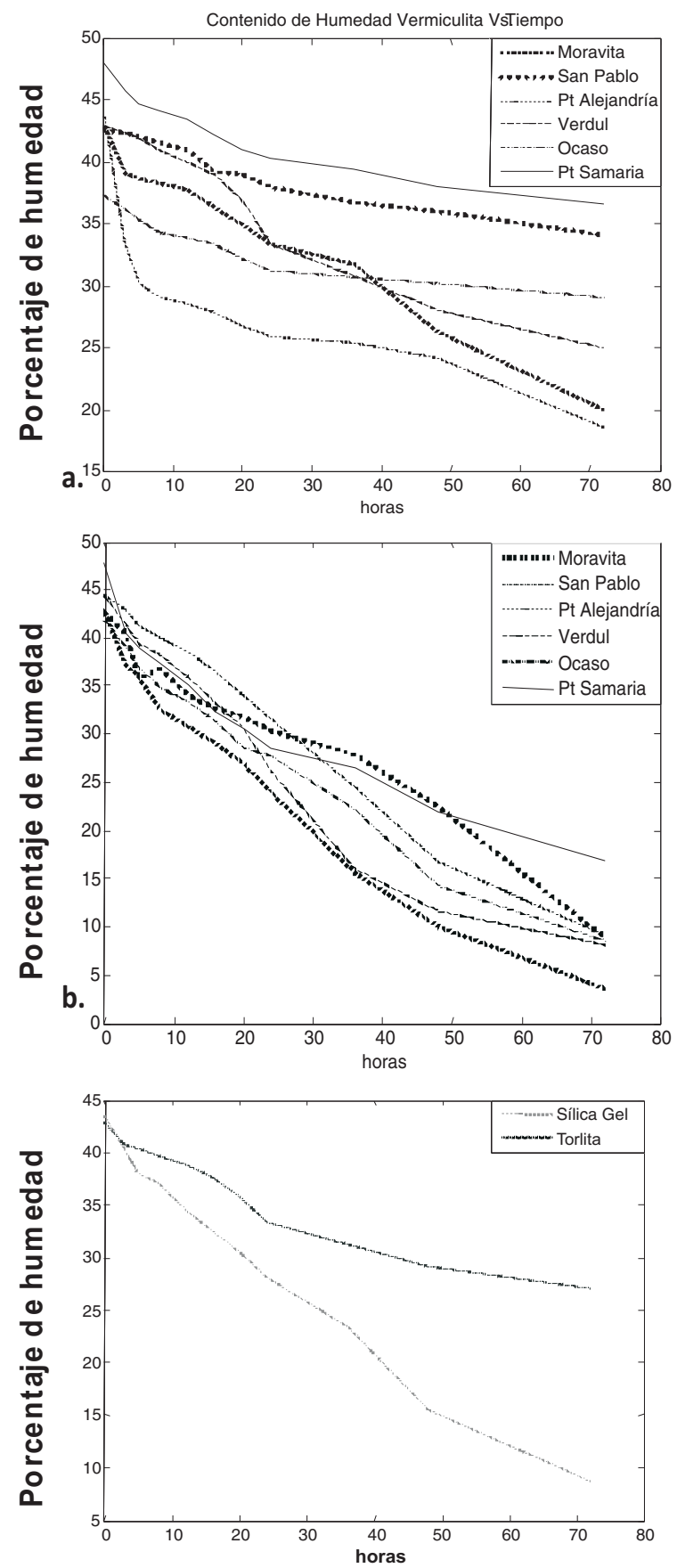

Figura 2. CH inicial de G. americana obtenido por los métodos de secado de sílica gel y torlita para los seis sitios de procedencia de las semillas. a. Secado con sílica gel. b. Secado con vermiculita. c. CH promedio.

\section{Viabilidad}

El PV inicial promedio obtenido por el método de Tinción con Tetrazolio para las semillas de G. americana fue de $75 \%$

(Figura 3). Se presentaron los mayores valores de viabilidad en las semillas procedentes de San Pablo, Ocaso y Puerto Samaria. Esta prueba concordó con los porcentajes de germinación iniciales $(75 \%$ de viabilidad y $78 \%$ de germinación).

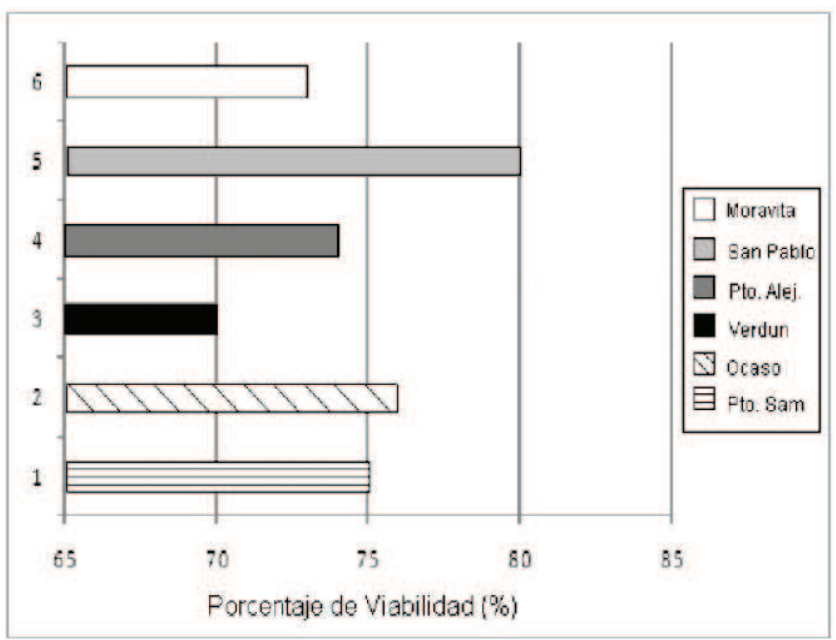

Figura 3. Porcentaje de viabilidad de G. americana para los seis sitios de procedencia de las semillas.

Entre los problemas con esta especie, se ha reportado la rápida pérdida de viabilidad de sus semillas, lo que ha obligado a la siembra después de la remoción de sus frutos, a fin de obtener los mejores resultados de germinación (16).

\section{Germinación}

EI PG inicial obtenido fue de $78.33 \%$ durante un periodo de 33 días; la TG promedio fue de 23 días y el IVG fue de 2 semillas por día (Figura 4).

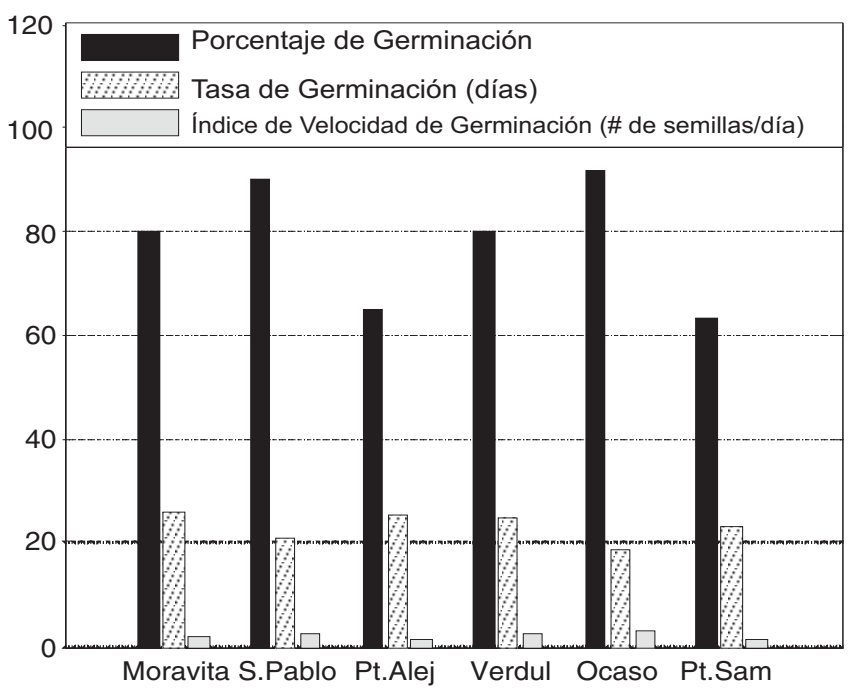

Figura 4. Prueba de Germinación Inicial de G. americana para los seis sitios de procedencia de las semillas

Las semillas que presentaron los mayores valores de PG 
fueron las procedentes del Ocaso: 91.67 y $90 \%$ respectivamente; así mismo tuvieron el mayor IVG de aproximadamente 3 semillas por día. Las mayores TG se presentaron en las semillas de La Moravita (26 días) y Puerto Alejandría (25 días).

EI PG y TG iniciales concuerdan con lo reportado por diversos autores en aspectos como el rango en el que se encuentra el porcentaje de germinación obtenido, el inicio de la germinación y el tiempo de finalización de la misma ${ }^{(17-}$ ${ }^{18)}$; además reportes sobre el máximo de germinación obtenido a temperatura ambiente, con el sustrato papel secante y la exposición a la luz durante el día ${ }^{(14)}$.

La obtención de altos porcentajes de germinación de semillas de $G$. americana con prealmacenamiento a contenidos de humedad entre 47 y $19 \%$, en el secado con torlita y decreciendo por debajo del $50 \%$ en el secado con sílica gel a un contenido de humedad menor a $9 \%$, indica que requieren de humedad superior al $10 \%$ para su germinación.

\section{Pruebas de almacenamiento}

Se obtuvieron valores entre 6.03 y $46.63 \%$ de $\mathrm{CH}$ (Tabla 6). El ANOVA del $\mathrm{CH}$ muestra que existen diferencias significativas para los factores: sustrato y tiempo de almacenamiento (Tabla 2, figura 5).

Los porcentajes más altos de $\mathrm{CH}$ para las semillas se presentaron almacenándolas en torlita con temperaturas

Tabla 2. ANOVA para el $\mathrm{CH}$ de semillas de $\mathrm{G}$. americana

\begin{tabular}{|c|c|c|c|c|c|}
\hline Fuente & $\begin{array}{c}\text { Suma de } \\
\text { cuadrados }\end{array}$ & GI & $\begin{array}{c}\text { Cuadrado } \\
\text { Medio }\end{array}$ & F-Ratio & P-Valor \\
\hline Procedenc & 5 & 2633.8 & 526.76 & & \\
\hline Sustrato & 1 & 4629.4 & 4629.44 & 96.80 & $0.0000^{\circ}$ \\
\hline Temperatu & 2 & 151.7 & 75.83 & 1.59 & 0.2093 \\
\hline Tiempo & 3 & 700.4 & 233.46 & 4.88 & $0.0031^{\circ}$ \\
\hline Sustrato*Temperatu & 2 & 68.8 & 34.39 & 0.72 & 0.4893 \\
\hline Sustrato*Tiempo & 3 & 122.7 & 40.89 & 0.85 & 0.4667 \\
\hline Temperatu*Tiempo & 6 & 249.3 & 41.55 & 0.87 & 0.5202 \\
\hline Sustrato*Temperatu'Tiempo & $\begin{array}{c}6 \\
115\end{array}$ & 240.7 & 40.12 & 0.84 & 0.5424 \\
\hline $\begin{array}{l}\text { Residual } \\
\text { Total }\end{array}$ & $\begin{array}{l}115 \\
143\end{array}$ & $\begin{array}{c}5499.8 \\
14296.6\end{array}$ & 47.82 & & \\
\hline
\end{tabular}

* Significativo al $0.95 \%$

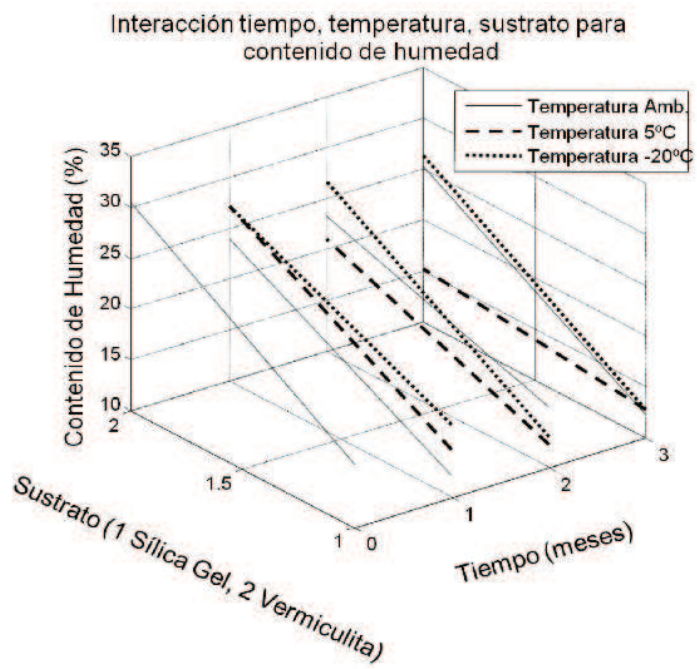

Figura 5. Interacción de 20 orden entre 3 factores: Tiempo, sustrato y Temperatura para el CH de semillas de $\mathbf{G}$. americana

La prueba de DMS muestra que los grupos $A$ y $B$ son significativamente diferentes uno del otro, siendo A el grupo correspondiente a un $\mathrm{CH}$ promedio de $26 \%$ obtenido con torlita y $\mathrm{B}$ el grupo con un $\mathrm{CH}$ de $14 \%$ obtenido por secado con sílica gel (Tabla 3).
Tabla 3. DMS para la comparación de medias de factores para el $\mathrm{CH}$ de semillas de $\mathbf{G}$. americana

\begin{tabular}{lcc}
\hline \multicolumn{1}{c}{ Factores } & Media & $\begin{array}{c}\text { Grupos } \\
\text { homogéneos }\end{array}$ \\
\hline $\begin{array}{l}\text { Temperatura } \\
\text { Ambiente }\end{array}$ & 19.836 & $\mathrm{~A}$ \\
$\mathbf{5}^{\circ} \mathrm{C}$ & 18.728 & $\mathrm{~A}$ \\
$-20^{\circ} \mathrm{C}$ & 21.241 & $\mathrm{~A}$ \\
Sustrato & 14.298 & $\mathrm{~B}^{*}$ \\
Silica gel & 25.638 & $\mathrm{~A}^{*}$ \\
Torlita & & \\
Tiempo & 23.313 & $\mathrm{~A}$ \\
$\mathbf{0}$ & 20.422 & $\mathrm{AB}$ \\
$\mathbf{1}$ & 18.720 & $\mathrm{~B}$ \\
$\mathbf{2}$ & 17.418 & $\mathrm{~B}$ \\
$\mathbf{3}$ & & \\
\hline
\end{tabular}

*Diferencias significativas al $0.95 \%$

El secado de semillas de la especie a un $\mathrm{CH}$ crítico entre 9-6\% resulta en decrecimiento en $\mathrm{PG}$, al igual que en $\mathrm{CH}$ entre 38$42 \%$. Mientras que cuando se almacena a temperatura ambiente a $11 \%$ de contenido de humedad la germinación se mantiene ${ }^{(15)}$.

De acuerdo a lo anterior, el bajo PG registrado en semillas almacenadas a 5-C pudo deberse a contenidos de humedad, menores o muy superiores al $11 \%$, considerado como óptimo para su conservación.

Por otra parte, se ha reportado que en el secado de las semillas que toleran desecación parcial deben considerarse, 
además del grado más pequeño de humedad segura, el grado de humedad crítico y el grado de humedad letal para cada especie; ya que puede ocurrir sensibilidad a la desecación entre diferentes lotes de la misma especie ${ }^{(19)}$.

El CH promedio de las semillas luego del secado, alcanzó aproximadamente el $10 \%$ el cual es considerado adecuado para que las semillas se conserven viables al menos a corto plazo.
En la prueba de viabilidad se obtuvieron valores entre el $10 \%$ y el $80 \%$ de PV, con un promedio de $42 \%$. Para las semillas almacenadas a 5 y -20 ㄷ C hubo dificultad en la evaluación de las estructuras del embrión por la coloración poco intensa de los tejidos a una concentración de tetrazolio al $0.1 \%$. EI ANOVA para el PV exhibe diferencias significativas para los factores Temperatura y Tiempo y para las interacciones de primer orden: Sustrato-Temperatura y Temperatura-Tiempo (Tabla 4).

Tabla 4. ANOVA para el PV de semillas de G. americana.

\begin{tabular}{|c|c|c|c|c|c|}
\hline Fuente & $\begin{array}{c}\text { Suma de } \\
\text { cuadrados }\end{array}$ & GI & $\begin{array}{c}\text { Cuadrado } \\
\text { Medio }\end{array}$ & F-Ratio & P-Valor \\
\hline Procedencia & 5 & 6070.2 & 1214.0 & & \\
\hline Sustrato & 1 & 25.0 & 25.0 & 0.09 & 0.7698 \\
\hline Temperatura & 2 & 7202.4 & 3601.2 & 12.40 & $0.0000^{*}$ \\
\hline Tiempo & 3 & 36334.8 & 12111.6 & 41.70 & $0.0000^{\circ}$ \\
\hline Sustrato*Temperatura & 2 & 2694.8 & 1347.4 & 4.64 & $0.0115^{\prime}$ \\
\hline Sustrato*Tiempo & 3 & 559.7 & 186.6 & 0.64 & 0.5893 \\
\hline Temperatura*Tiempo & 6 & 4551.7 & 758.6 & 2.61 & $0.0207^{\prime}$ \\
\hline Sustrato*Temperatura*Tiempo & 6 & 3470.5 & 578.4 & 1.99 & 0.0725 \\
\hline Residual & 115 & 33403.4 & 290.5 & & \\
\hline Total & 143 & 94312.6 & & & \\
\hline
\end{tabular}

*Significativo al $0.95 \%$.

La viabilidad de las semillas es mayor para el almacenamiento a temperatura ambiente en torlita; la menor viabilidad se registró en el almacenamiento a -20 ㅇ C de temperatura y se evidencia como va disminuyendo a medida que transcurre el tiempo (Figura 6).

Se ha registrado que almacenadas en condiciones ambientales las semillas de G. americana conservan su viabilidad por dos meses ${ }^{(17)}$, lo que se asemeja con los resultados obtenidos.

Se puede inferir que la combinación de temperatura de 5으 con alto $\mathrm{CH}$ deteriora la calidad de las semillas almacenadas

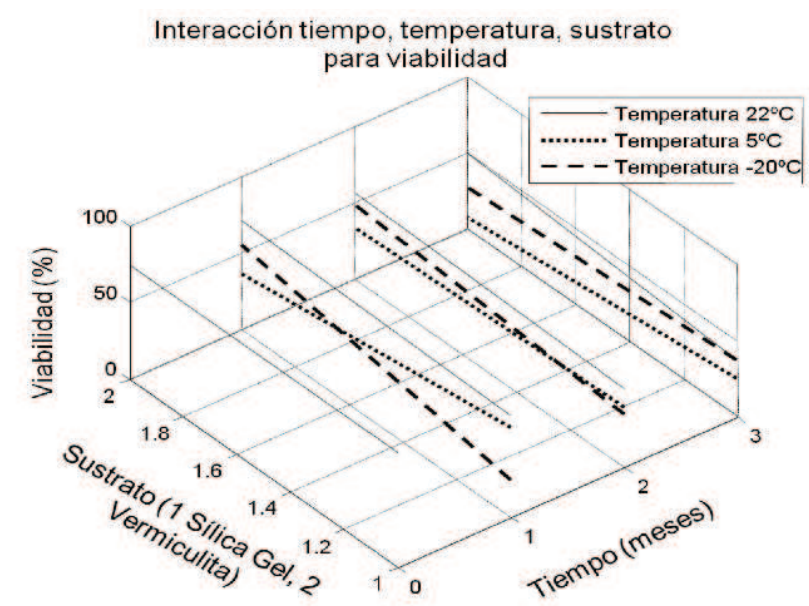

Figura 6. Interacción de 20 orden entre 3 factores: Tiempo, sustrato y temperatura para el PV de semillas de G. americana. y que las semillas son sensibles a bajas temperaturas; pues en esta investigación la viabilidad obtenida en almacenamiento a -20 oc fue inferior al $50 \%$.

Para las semillas almacenadas a 5 y -20 -C hubo dificultad en la evaluación de las estructuras del embrión por la coloración poco intensa de los tejidos a una concentración de tetrazolio al $0.1 \%$.

De acuerdo a lo anterior, la baja viabilidad de muchas semillas y la poca concordancia de ésta prueba con la de germinación pudo deberse a la concentración de tetrazolio, que quizá estuvo muy alta para la especie; ya que existen registros de mejores patrones de tinción cuando se utilizan concentraciones de $0.05 \%$ en vez de $1 \%(16,20-21)$.

En la prueba de germinación se obtuvieron valores de PG entre $0-100 \%$; la TG varió entre 23 a 33 días y el IVG fue inferior a 1 semilla por día. El ANOVA para la variable PG, muestra diferencias significativas para los factores e interacciones entre factores (Tabla 5). 
Tabla 5. ANOVA para el PG de semillas de Genipa americana.

\begin{tabular}{lccccc}
\hline Fuente & $\begin{array}{c}\text { Suma de } \\
\text { cuadrados }\end{array}$ & Gl & $\begin{array}{c}\text { Cuadrado } \\
\text { Medio }\end{array}$ & F-Ratio & P-Valor \\
\hline Procedenc & 5 & 0.0834 & 0.01689 & & \\
Sustrato & 1 & 0.3772 & 0.37720 & 19.69 & $0.0000^{*}$ \\
Temperatu & 2 & 0.7544 & 0.37720 & 19.69 & $0.0000^{*}$ \\
Tiempo & 3 & 18.0245 & 6.00816 & 313.66 & $0.0000^{*}$ \\
Sustrato*Temperatu & 2 & 0.7544 & 0.37720 & $19.69^{*}$ & $0.0000^{*}$ \\
Sustrato*Tiempo & 3 & 0.2854 & 0.09512 & 4.97 & $0.0028^{*}$ \\
Temperatu*Tiempo & 6 & 0.5707 & 0.09512 & 4.97 & $0.0001^{*}$ \\
Sustrato*Temperatu*Tiempo & 6 & 0.5707 & 0.09512 & 4.97 & $0.0001^{*}$ \\
Residual & 115 & 2.2028 & 0.01915 & & \\
Total & 143 & 23.6235 & & & \\
\hline
\end{tabular}

*Significativo al $0.95 \%$.

Los porcentajes más altos de germinación para las semillas almacenadas se presentaron con torlita a temperatura ambiente (Figura 7).

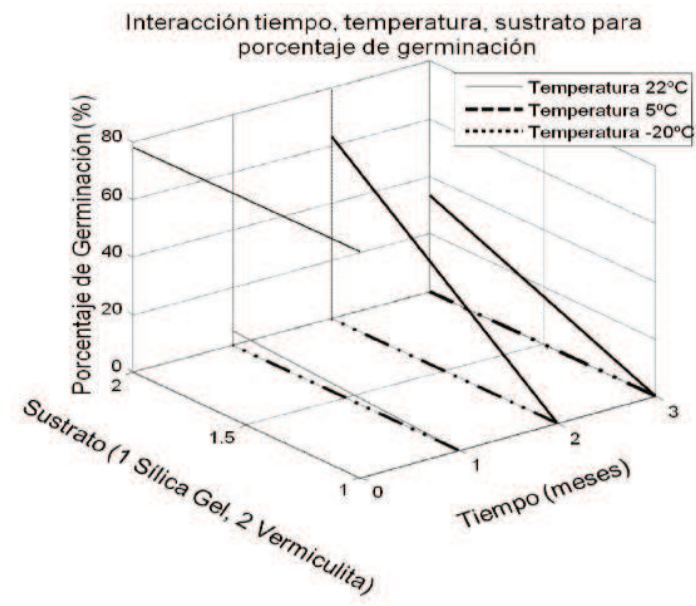

Figura 7. Interacción de 20 orden entre 3 factores: Tiempo, sustrato y temperatura de almacenamiento para el PG de semillas de G. americana.
La prueba de DMS para el PG de las semillas evidencia que los grupos A y B son significativamente diferentes uno del otro, siendo A el correspondiente al $28 \%$ de PG después del almacenamiento con torlita y B el $20 \%$ de PG obtenido con sílica gel (Tabla 6).

Tabla 6. DMS para la interacción de factores en el PG de semillas de G. americana.

\begin{tabular}{ccc}
\hline Factores & Media & Grupos homogéneos \\
\hline Temperatura & & \\
Ambiente & 32.250 & A \\
$5^{\circ} \mathrm{C}$ & 19.542 & B \\
$-20^{\circ} \mathrm{C}$ & 19.542 & $\mathrm{~B}$ \\
Sustrato & & \\
Silica gel & 19.542 & $\mathbf{B}^{\circ}$ \\
Torlita & 28.041 & $\mathrm{~A}^{*}$ \\
Tiempo & 78.167 & $\mathrm{~A}$ \\
$\mathbf{0}$ & 0.833 & $\mathrm{C}$ \\
1 & 10.556 & $\mathrm{~B}$ \\
$\mathbf{2}$ & 5.556 & $\mathrm{BC}$ \\
\hline
\end{tabular}

*Diferencias significativas al $0.95 \%$

EI ANOVA de la TG reveló diferencias significativas para todos los factores e interacciones entre éstos (Tabla 7).

Tabla 7. ANOVA para la TG de semillas de G. americana.

\begin{tabular}{|c|c|c|c|c|c|}
\hline Fuente & $\begin{array}{c}\text { Suma de } \\
\text { cuadrados }\end{array}$ & GI & $\begin{array}{c}\text { Cuadrado } \\
\text { Medio }\end{array}$ & F-Ratio & P-Valor \\
\hline Procedencia & 5 & 2.439 & 0.4877 & & \\
\hline Sustrato & 1 & 7.664 & 7.6637 & 22.78 & $0.0000^{\circ}$ \\
\hline Temperatura & 2 & 15.327 & 7.6637 & 22.78 & $0.0000^{\circ}$ \\
\hline Tiempo & 3 & 217.529 & 72.5096 & 215.53 & $0.0000^{*}$ \\
\hline Sustrato*Temperatura & 2 & 15.327 & 7.6637 & 22.78 & $0.0000^{\circ}$ \\
\hline Sustrato*Tiempo & 3 & 3.984 & 1.3281 & 3.95 & $0.0101^{*}$ \\
\hline Temperatura'Tiempo & 6 & 7.969 & 1.3281 & 3.95 & $0.0013^{\circ}$ \\
\hline Sustrato*Temperatura'Tiempo & 6 & 7.969 & 1.3281 & 3.95 & $0.0013^{\circ}$ \\
\hline Residual & 115 & 38.688 & 0.3364 & & \\
\hline Total & 143 & 316.896 & & & \\
\hline
\end{tabular}

*Significativo al $0.95 \%$. 
La TG es mayor cuando se almacena con torlita a temperatura ambiente (Figura 8).

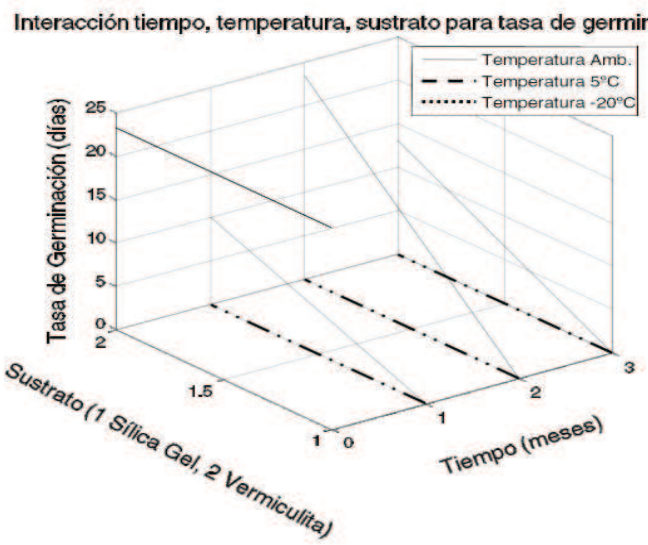

Figura 8. Interacción de 20 orden entre 3 factores:

Tiempo, sustrato y temperatura de almacenamiento para la TG de semillas de $G$. americana.

La prueba de DMS para la TG de las semillas muestra que los grupos A y B son significativamente diferentes uno del otro, siendo A el valor promedio de tasa de germinación de 31 días para silica Gel y 29 días para torlita (Tabla 8).

Tabla 8. DMS para la interacción de factores en la TG de semillas de G. americana.

\begin{tabular}{|c|c|c|}
\hline Factores & Media & Grupos homogéneos \\
\hline \multicolumn{3}{|l|}{ Temperatura } \\
\hline $\begin{array}{c}\text { Ambiente } \\
5^{\circ} \mathrm{C} \\
-20^{\circ} \mathrm{C} \\
\text { Sustrato }\end{array}$ & $\begin{array}{l}31 \\
31 \\
29\end{array}$ & $\begin{array}{l}A \\
A \\
B\end{array}$ \\
\hline $\begin{array}{l}\text { Silica gel } \\
\text { Torlita }\end{array}$ & $\begin{array}{l}31 \\
29\end{array}$ & $\begin{array}{l}A^{\prime} \\
B^{\prime}\end{array}$ \\
\hline \multicolumn{3}{|l|}{ Tiempo } \\
\hline 0 & 22.687 & B \\
\hline 1 & 33 & A \\
\hline 2 & 29 & A \\
\hline 3 & 30 & A \\
\hline
\end{tabular}

*Diferencias significativas a

EI ANOVA del IVG, muestra diferencias significativas para todos los factores y para la interacción de primer orden: Sustrato-Temperatura (Tabla 9). El IVG durante el almacenamiento presenta valores inferiores a 1 semilla por día (Figura 9).

Tabla 9. ANOVA para el ÍVG de semillas de G. americana.

\begin{tabular}{|c|c|c|c|c|c|}
\hline Fuente & $\begin{array}{l}\text { Suma de } \\
\text { cuadrados }\end{array}$ & GI & $\begin{array}{c}\text { Cuadrado } \\
\text { Medio }\end{array}$ & F-Ratio & P-Valor \\
\hline $\begin{array}{l}\text { Procedencia } \\
\text { Sustrato } \\
\text { Temperatura } \\
\text { Tiempo } \\
\text { Sustrato*Temperatura } \\
\text { Sustrato*Tiempo } \\
\text { Temperatura*Tiempo } \\
\text { Sustrato*Temperatura* Tiempo } \\
\text { Residual } \\
\text { Total }\end{array}$ & $\begin{array}{l}5 \\
1 \\
2 \\
3 \\
2 \\
3 \\
6 \\
6 \\
115 \\
143\end{array}$ & $\begin{array}{c}0.3006 \\
0.1111 \\
0.22222 \\
53.4344 \\
0.2222 \\
0.0744 \\
0.1487 \\
0.1487 \\
2.0811 \\
56.7235\end{array}$ & $\begin{array}{c}0.0601 \\
0.1111 \\
0.1111 \\
17.8115 \\
0.1111 \\
0.0248 \\
0.0248 \\
0.0248 \\
0.0179\end{array}$ & $\begin{array}{c}6.20 \\
6.20 \\
993.78 \\
6.20 \\
1.38 \\
1.38 \\
1.38\end{array}$ & $\begin{array}{l}0.0142^{\prime} \\
0.0028^{\prime} \\
0.0000^{\prime} \\
0.0028^{\prime} \\
0.2515 \\
0.2272 \\
0.2272\end{array}$ \\
\hline
\end{tabular}

*Significativo al $0.95 \%$.

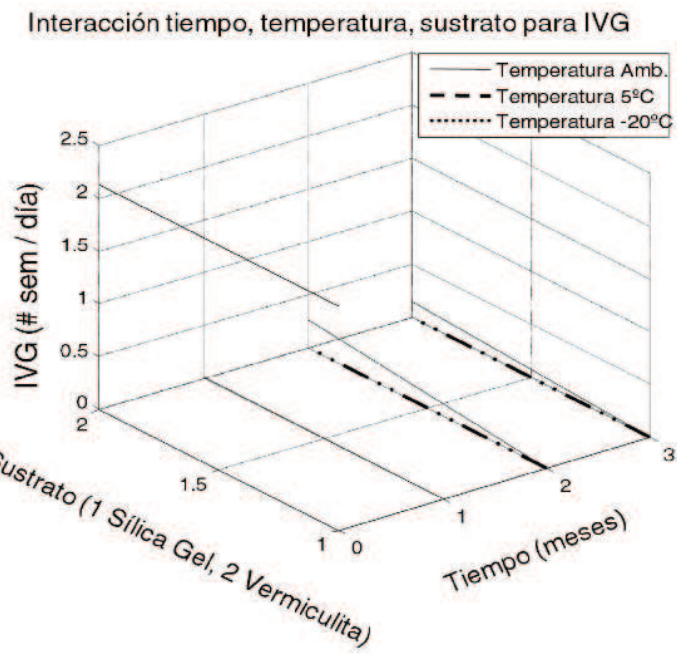

Figura 9. Interacción de 20 orden entre 3 factores: Tiempo, sustrato y temperatura de almacenamiento para el IVG de semillas de G. americana.
En general, las semillas presentaron alta contaminación fúngica asociada a la humedad en el almacenamiento, ya que las semillas almacenadas a baja temperatura con el sustrato torlita aumentaron su humedad interna, elevándose el ataque fúngico. Para el caso del sustrato sílica gel, el $\mathrm{CH}$ estuvo por debajo del $10 \%$ en varios casos, por lo que la viabilidad de las semillas se redujo y no germinaron.

La combinación de tratamientos sustrato-temperatura de almacenamiento en los que hubo germinación de semillas fue torlita a temperatura ambiente y sólo para este caso la contaminación por hongos fue escasa.

Se ha mencionado que la temperatura óptima para germinación de las semillas de G. americana extraídas de frutos inmaduros y de frutos maduros se encuentra en la franja de $22^{\circ} \mathrm{C}$ a $31^{\circ} \mathrm{C}^{(21)}$.

La germinación durante el almacenamiento fue nula a -20ㄷ, lo que indica que las semillas son sensibles a la conservación a esta temperatura. Resultados similares fueron obtenidos por otros autores ${ }^{(15)}$. 
En general la germinación se vio afectada por la contaminación fúngica y daños internos de la semilla almacenada, no visibles a simple vista previo al inicio de la germinación.

Con respecto a lo anterior, se ha reportado que existe ocurrencia de hongos y es independiente de la procedencia de los frutos; además, la mayoría de los hongos patógenos está asociada a las semillas procedentes de frutos maduros y pueden afectar interna o externamente la semilla ${ }^{(14-15)}$.

La incidencia de hongos xerotolerantes y de aquellos de campo en semillas $G$. americana puede ser atribuida a contaminación por transmisión sistémica de la planta madre y vía estigma durante la floración y su localización en tejidos intra-seminales ${ }^{(15)}$. Lo anterior debido a que si los hongos se presentaran externamente, la desinfestación superficial con hipoclorito de sodio, probablemente los habría eliminado.

Para G. americana las bajas temperaturas no son las adecuadas para los niveles máximos de su germinación, además provocan un ambiente propicio para la proliferación de hongos xerotolerantes.

Se ha reportado que las semillas de G. americana pueden ser parcialmente desecadas, sin embargo pierden gradualmente la viabilidad durante el almacenamiento a corto plazo en bajas temperaturas ${ }^{(14-15)}$, lo que se confirma en la presente investigación.

Los resultados obtenidos concuerdan con lo reportado en la literatura ${ }^{(15)}$, pues, en general, las semillas de G. americana se conservan mejor a temperatura ambiente en un $\mathrm{CH}$ por encima del $10 \%$ Existe una interacción entre la temperatura y el $\mathrm{CH}$ de la semilla; de ambos depende su buena conservación, ya que la temperatura influye en la absorción de humedad, durante el almacenamiento.

Por otra parte, con respecto al sustrato para control de humedad, se encontró que el mejor sustrato correspondió a la torlita, pues no permite que la humedad disminuya por debajo del contenido crítico (menos del 10\%).
Los resultados sugieren que las semillas de G. americana presentan comportamiento intermediario en el almacenamiento, soportando la desecación en contenidos de humedad próximos a $10 \%$ y no toleran el congelamiento; como lo mencionan algunos autores ${ }^{(14-21)}$.

\section{CONCLUSIONES}

$\checkmark$ El potencial germinativo de la especie puede mantenerse a corto plazo en almacenamiento a temperatura ambiente, utilizando torlita para controlar la humedad.

$\checkmark$ Las semillas almacenadas a baja temperatura y alto contenido de humedad son más vulnerables al deterioro por contaminación fúngica.

$\checkmark$ El comportamiento de almacenamiento de $G$. americana con respecto a la temperatura y el contenido de humedad corresponde al de una semilla intermedia.

$\checkmark$ Las semillas de G. americana deben almacenarse con un contenido de humedad por encima del $10 \%$ y en temperatura ambiente para conservar su viabilidad.

\section{AGRADECIMIENTOS}

Los autores del proyecto agradecen a:

- Centro del Estudios e Investigaciones en Biodiversidad y Biotecnología de la Universidad del Quindío (CIBUQ). Instalaciones, equipos, insumos y material de campo.

- Reserva Natural La montaña del Ocaso. Apoyo logístico.

- Jamid Escobar y Leider Suárez. Guardaselvas del Ocaso.

- M. Sc. Rocío Stella Suárez R y Asp. M. Sc. Lina Marcela Arbeláez Arias. Por su asesoría durante el desarrollo de este proyecto.

\section{BIBLIOGRAFÍA}

1. Vázquez-Yanes, C. \& M. Rojas, A. Ex Situ Conservation of Tropical Rain Forest Seed: Problems and Perspectives. INTERCIENCIA 21(5). p. 293-298. 1996. URL: http://www.interciencia.org.ve.

2. Iriondo, A. J.M. Conservación de germoplasma de especies raras y amenazadas (Revisión). Invest. Agr.: Prod. Prot. Veg. Vol. 16 (1). 20 p.2001.

3. Jøker, D., DFSC, Salomão, N. A., Vasquez, C. Y W. Vásquez, CATIE. Genipa americana L. 2003. Seed Leaflet. No.67. 2003.

4. Mendoza, H., Ramírez, B. \& L.C. Jiménez. Rubiaceae de Colombia-Guía ilustrada de géneros. Instituto de Investigación de Recursos Biológicos Alexander Von Humboldt. Bogotá. Colombia. 351 p. 2004.

5. Andrade, A.C.S., Souza, A.F, Ramos, F.N., Pereira, T.S. \& A.P.M. Cruz. Germinação de sementes de Jenipapo: Temperatura, substrato e morfologia do desenvolvimento pós-seminal. Pesq. agropec. bras., Brasília, v.35, n.3. p.609-615. 2000.

6. Arias, V.A, Gómez, G. \& C. Agudelo. En: Riqueza Biótica Quindiana. Compilado por C.H. Agudelo, H. Editorial Universidad del Quindío, 421 p. 2006.

7. Orozco, C. A. F \& G. D. Gómez. Fenología de especies forestales En: Riqueza Biótica Quindiana, Capitulo II Editorial Universidad del Quindío. 2006. 421p. En: Riqueza Biótica Quindiana.

8. Orozco, C. A. F., Arias V.A. \& L.G. Mejía. Fenología de Anacardium excelsum, Rollinia membranacea y Genipa americana en la Reserva del Ocaso, Quimbaya - Quindío. Trabajo de Grado. (Licenciatura en Biología) Universidad del Quindío. Facultad de Ciencias Básicas y Tecnologías. Programa de Biología y Educación Ambiental. 117 p. 2002.

9. Oficina para la Coordinación de Asuntos Humanitarios / Naciones Unidas Colombia. OCHA. [en línea] Página Web versión HTML. Bogotá, Colombia: (s.e.), 2006. [citado 12 de Agosto de 2009]. Disponible en Internet: <http://www.colombiassh.org/site/spip.php?article27>. 
10. Rao, N.K., J. Hanson, M.E. Dulloo, K. Ghosh, D. Novell \& M. Larinde. Manual para el manejo de semillas en bancos de germoplasma. Manuales para Bancos de Germoplasma No. 8. Bioversity International, Roma, Italia. 165 p. 2007.

11. Maguire, J. Speed of germination - Aid in selection and evaluation for seedling emergency and vigor. Crop Science 2:176. 1962.

12. Gómez, T.J., Jasso, M.J., Vargas, H.J.V. \& M.R. Soto H. Deterioro de semilla de dos procedencias de Swietenia macrophylla King., bajo distintos métodos de almacenamiento. Ra simhai, enero-abril, vol 2 (001). Universidad Autónoma Indígena de México. El Fuerte México. p. 223-239. 2006.

13. Gómez, C.C. A guide to efficient long term seed preservation.Monographs ETSIA, Univ. Politécnica de Madrid 170. p. 1-17. 2007.

14. Salomão, A.N. Desiccation, storage and germination of Genipa Americana seeds. En: Sacandé, M., D. Joker, M.E. Dulloo \& K.A. Thomsen. Comparative Storage Biology of Tropical Tree Seeds. International Plant Genetic Resources Institute, Rome, Italy. 363 p. 2004.

15. Salomão, A.N. \& L. S. Padilha. Avaliação preliminar da germinabilidade e da micoflora associada às sementes de Genipa americana em diferentes estágios de maturação. Brasília: Embrapa Recursos Genéticos e Biotecnologia, (Série Embrapa Circular Técnica 50). 9 p. 2006.

16. Nascimento, W.M.O. \& N. M. Carvalho. Determinação da viabilidade de sementes de jenipapo (Genipa americana L.) através do teste de tetrazólio. Revista Brasileira de Sementes, Campinas, v.20, n.2. p. 470-474. 1998.

17. Salazar-Figueroa, R. Genipa americana Linnaeus. Nota Técnica sobre Manejo de Semillas Forestales CATIE no. 72. Editorial Turrialba, Centro Agronómico Tropical de Investigación y Enseñanza (CATIE), CR. 2 p. 1999.

18. Agudelo, H.C.A, Orozco, C.A.F \& G.D. Gómez M. Banco de Germoplasma de especies forestales del departamento del Quindío. Revista de Investigaciones No. 16. Universidad del Quindío. Armenia.p 81-92. 2006

19. Fonseca, S.C. L. \& H. B. Freire. Sementes Recalcitrantes: Problemas na pós colheita. Artigo de revisäo. Bragantia, Campinas, v.62, n.2. p. 297-303. 2003.

20. Ferreira, R.A., Oliveira, L.M., Tonetti, O.A.O. \& A.C. Davide. Comparação da viabilidade de sementes de Schizolobium parahyba (VELL.) Blake - Leguminosae Caesalpinoideae, pelos testes de germinação e tetrazólio. Revista Brasileira de Sementes, vol. 29, no 3. p.83-89. 2007.

21. Silva, D.B., Salomão, A.N., Caravalho. P.C.L. \& M.M.V.S. Wetzel. Jenipapo. En: Vieira, R. F. (ed.) Frutas nativas da região Centro-Oeste do Brasil. Brasília: Embrapa Recursos Genéticos e Biotecnologia. p. 304-322. 2006.

22. Acero, F.P.A. Análisis y Ensayos de Almacenamiento para semillas de Hymenaea courbaril y Enterolobium cyclocarpum del Quindío. Trabajo de grado (Licenciado en Biología). Universidad del Quindío. Facultad de Ciencias Básicas y Tecnologías. Programa de Licenciatura en Biología.150 p. 2005.

23. Vázquez, Y.C., Orozco, A., Rojas, M., Sánchez, M.E. \& V.Cervantes. La reproducción de las plantas: Semillas y Meristemos. La ciencia para todos, № 157. Fondo de Cultura Económica. México, D.F. 1ạ ed. 166 p. 1997. 